\title{
NOTA
}

\section{COMPARAÇÃO ENTRE SOLOS SOB USO AGROFLORESTAL E EM FLORESTAS REMANESCENTES ADJACENTES, NO NORTE DE RONDÔNIA ${ }^{(1)}$}

\author{
José Maria Thomaz Menezes ${ }^{(2)}$, Johannes van Leeuwen ${ }^{(2)}$, Sérgio \\ Valiengo Valeri ${ }^{(3)}$, Mara Cristina Pessôa da Cruz ${ }^{(3)}$ \& Raimundo \\ Cajueiro Leandro ${ }^{(2)}$
}

\begin{abstract}
RESUMO
A importância e a eficácia dos sistemas agroflorestais na conservação dos solos tropicais necessitam de comprovações demonstrativas localizadas. Atributos físicos e químicos dos solos de sete parcelas com cinco anos e meio de uso agroflorestal, avaliados na camada de $0-0,2 \mathrm{~m}$, foram comparados aos respectivos solos de florestas remanescentes adjacentes no norte do Estado de Rondônia. Não foram constatadas diferenças na composição granulométrica entre os solos na camada avaliada, sugerindo que os dois sistemas localizam-se sob o mesmo tipo de solo numa mesma propriedade rural. Nos solos sob sistemas agroflorestais, os valores de $\mathrm{pH}$, Ca e Mg foram sempre maiores do que os dos solos das florestas remanescentes adjacentes, o que é atribuído ao aporte de nutrientes que restaram da queima anterior da floresta, ocorrida entre um e oito anos antes da instalação do sistema agroflorestal. Os teores de matéria orgânica, P e K do solo nos sistemas agroflorestais mantiveram-se com valores semelhantes aos da floresta, cinco anos e meio depois de sua instalação, indicando que esses sistemas preservaram algumas propriedades químicas do solo durante o período considerado.
\end{abstract}

Termos de indexação: conservação do solo, sistemas agroflorestais, solos tropicais.

\footnotetext{
(1) Trabalho extraído da Tese de Doutorado do primeiro autor, programa de Pós-graduação em Produção Vegetal da Faculdade de Ciências Agrárias e Veterinárias, Campus de Jaboticabal - FCAV-UNESP. Apoio financeiro do CNPq. Recebido para publicação em julho de 2006 e aprovado em novembro de 2007.

(2) Pesquisador do Instituto Nacional de Pesquisas da Amazônia - INPA, CPCA. Av. André Araújo 2936, CEP 69060-001 Manaus (AM). E-mails: jmtm@inpa.gov.br; leeuwen@inpa.gov.br; caju@inpa.gov.br

(3) Professor da Faculdade de Ciências Agrárias e Veterinárias, Universidade Estadual Paulista - FCAV/UNESP. Rod. Paulo Donato Castellane s/n, CEP 14884-900 Jaboticabal (SP).E-mail: valeri@fcav.unesp.br; mcpcruz@fcav.unesp.br
} 


\title{
SUMMARY: COMPARISON OF SOILS USED FOR AGROFORESTRY AND OF REMAINING FORESTS, INNORTHERNRONDÔNIA STATE, BRAZIL
}

\begin{abstract}
The importance and efficiency of agroforestry systems in the conservation of tropical soils need to be demonstrated at the local scale. Physical and chemical soil attributes of seven plots used for agroforestry during 5.5 years were evaluated in the $0-0.2 \mathrm{~m}$ layer and compared with the respective soils of adjacent forests fragments in the Northern Rondônia State. No differences in texture were found between the soils in the layer evaluated, indicating that the two systems are located on the same soil type. In the soils under agroforestry systems, the values of $\mathrm{pH}$, calcium and magnesium were always higher than in the soils of the adjacent forest fragments, which are ascribed to the nutrients released by the previous forest slashing and burning, between one and eight years before the installation of the agroforestry systems. The contents of soil organic matter, phosphorus and potassium in the 5.5 years after the installation of the agroforestry systems remained at values similar to those of the forest fragments. These results indicate that the agroforestry systems preserved some chemical soil properties at similar levels as the natural forest in the study period.
\end{abstract}

Index terms: soil conservation, agroforestry systems, tropical soils.

\section{INTRODUÇÃO}

Os atributos do solo sob uso agrícola são, normalmente, influenciados pelo manejo das culturas. O controle pouco intensivo da vegetação espontânea favorece o crescimento e desenvolvimento de capoeiras, que, se manejadas adequadamente, podem ser benéficas para a recuperação e manutenção da fertilidade dos solos, sobretudo nas regiões tropicais.

A plantação de sistemas agroflorestais (SAFs) tem emergido como uma alternativa de uso da terra para pequenos agricultores da América Tropical, devido ao seu potencial de reduzir a degradação do solo e diminuir a pressão sobre as áreas de florestas (Smith et al., 1998; Mcgrath et al., 2000). Os SAFs caracterizam-se pela combinação de espécies arbóreas com culturas agrícolas e, ou, com animais domésticos, simultaneamente, ou alternados no tempo e no espaço (Dubois at al., 1996). Na Amazônia, o grande desafio tem sido desenvolver sistemas capazes de conciliar, de forma harmoniosa, interesses de conservação ambiental com sustentabilidade econômica, em substituição à agricultura migratória comumente praticada.

Para poder formular propostas para SAFs que garantam sustentabilidade para os agricultores, o Núcleo Agroflorestal do INPA acompanha, em alguns municípios no norte do Estado de Rondônia, um conjunto de sistemas agroflorestais piloto instalados em áreas de agricultores (van Leeuwen, 2002). Este trabalho compara os atributos físicos e químicos do solo de SAFs com os do solo de áreas adjacentes em que permaneceu a floresta natural.

\section{MATERIAL E MÉTODOS}

O estudo foi realizado no município de Itapuã do Oeste, situado no norte do Estado de Rondônia, numa área abrangida pelas coordenadas geográficas entre $9^{\circ} 02^{\prime} 40^{\prime \prime S}$ e $9^{\circ} 17^{\prime} 25^{\prime \prime S ~} \mathrm{~S}$ e $63^{\circ} 06^{\prime} 46^{\prime} \mathrm{W}$ e $63^{\circ} 28^{\prime} 33^{\prime}$ W. O clima é caracterizado como tropical chuvoso do tipo Am, segundo a classificação de Köppen (Bastos \& Diniz, 1982). Os plantios foram instalados em fevereiro de 1996 (época chuvosa 1995/96) em sete pequenas propriedades rurais do referido município, sendo planejados de acordo com a disponibilidade da área em cada propriedade e interesse de cada proprietário (van Leeuwen, 2002). Assim, houve grande variação entre os SAFs quanto à composição das espécies e o tamanho das áreas (Quadro 1).

As espécies predominantes nos plantios foram: açaí (Euterpe oleracea Martius), pupunha (Bactris gasipaes Kunth), ipê-roxo (Tabebuia sp), freijó-louro (Cordia alliodora Ruiz \& Pavon), bandarra (Schizolobium amazonicum Ducke.), cedro-rosa (Cedrela odorata L.), mogno (Swietenia macrophylla King) e teca (Tectona grandis L.F.). O tamanho das áreas plantadas variou de 0,5 a 1,90 ha. As árvores foram plantadas com espaçamento de $5 \times 5 \mathrm{~m}$. Todos os plantios foram feitos em áreas de relevo plano ou suavemente ondulados, em solos predominantemente do tipo Latossolo Vermelho-Amarelo. Seis das sete áreas dos SAFs foram inicialmente derrubadas para a produção de culturas anuais, um a oito anos antes da instalação do SAF (Quadro 1). Em apenas um caso, o plantio agroflorestal foi feito no meio da lavoura da mandioca. Nos demais, não houve mais plantios de culturas anuais após a implantação dos plantios agroflorestais. Em apenas um dos plantios, houve queima como conseqüência do preparo da área cuja vegetação era de capoeira alta (Quadro 1). No preparo das áreas para os outros seis plantios, não houve necessidade de queima, já que estes sucederam as culturas anuais do último ano agrícola, havendo apenas necessidade de roçagem da capoeira baixa de formação recente. Não houve aplicação de nenhum fertilizante em todas as áreas antes ou após a implantação dos SAFs. 
Quadro 1. Identificação, tamanho da área de plantio, ano do início da exploração (derrubada) e histórico do uso das áreas plantadas nos sete sistemas agroflorestais (SAFs)

\begin{tabular}{|c|c|c|c|}
\hline $\begin{array}{l}\text { Identificação } \\
\text { dos SAFs } \\
(1)\end{array}$ & $\begin{array}{l}\text { Tamanho } \\
\text { da área }\end{array}$ & $\begin{array}{l}\text { Ano de início da } \\
\text { exploração (verão) }\end{array}$ & Uso anterior da área \\
\hline & ha & & \\
\hline $\mathrm{IO}-4 \mathrm{~A}$ & 0,84 & 1992 & $\begin{array}{l}\text { Cultura anual: milho (2 anos). Em seguida, houve } \\
\text { formação da capoeira baixa seguida de roçagem, sem } \\
\text { queima, para o plantio do SAF. }\end{array}$ \\
\hline IO-6 & 0,56 & 1991 & $\begin{array}{l}\text { Culturas anuais: arroz, feijão e milho (2 anos); cana-de- } \\
\text { açúcar (1 ano). Em seguida, formação de capoeira baixa e } \\
\text { seu roço, sem queima, para plantio do SAF. }\end{array}$ \\
\hline $\mathrm{IO}-21 \mathrm{~A}$ & 0,57 & 1992 & $\begin{array}{l}\text { Culturas anuais: arroz e mandioca ( } 2 \text { anos). Depois, } \\
\text { formação da capoeira baixa e seu roço, sem queima, para } \\
\text { plantio do SAF. }\end{array}$ \\
\hline $\mathrm{IO}-34 \mathrm{~A}$ & 0,55 & 1988 & $\begin{array}{l}\text { Formação de capoeira alta de até } 5 \text { anos, depois } \\
\text { derrubada e queima. Em seguida, plantio de milho, arroz } \\
\text { e mandioca, até retirada para plantio do SAF. }\end{array}$ \\
\hline $\mathrm{IO}-41 \mathrm{~A}$ & 0,57 & 1995 & $\begin{array}{l}\text { Mandioca no primeiro ano. O plantio foi feito pouco antes } \\
\text { da colheita da mandioca. }\end{array}$ \\
\hline $\mathrm{IO}-42 \mathrm{~A}$ & 1,20 & 1988 & $\begin{array}{l}\text { Consórcio banana x cupuaçu ( } 4 \text { anos). Depois da retirada } \\
\text { das bananeiras, permaneceu só o cupuaçu nos anos } \\
\text { seguintes. As árvores do SAF foram plantadas nas } \\
\text { entrelinhas dessa cultura. }\end{array}$ \\
\hline IO-44 & 0,57 & 1992 & $\begin{array}{l}\text { Culturas anuais: arroz e milho (primeiro ano). Em } \\
\text { seguida, formação da capoeira alta seguida de seu roço e } \\
\text { queima, para plantio do SAF. }\end{array}$ \\
\hline
\end{tabular}

(1) Os SAFs foram codificados com as letras iniciais do município onde foram implantados (IO = Itapuã do Oeste) seguidas do número de identificação do plantio. A última letra do código (A) identifica o sistema agroflorestal (SAF) dentro da propriedade que possui diferentes unidades de SAFs.

Adjacente a cada SAF encontrava-se, ainda, uma área onde a floresta original foi mantida como reserva legal. Em cada uma das áreas dos SAFs e das respectivas florestas adjacentes, foi coletada uma amostra de solo composta por vinte amostras simples, numa profundidade de 0 a $20 \mathrm{~cm}$, em agosto de 2001 . $\mathrm{O}$ procedimento da coleta na floresta original foi realizado mediante a eliminação da serrapilheira da superfície, seguida da retirada da amostra com auxílio de um trado tipo "holandês". As amostras foram analisadas fisicamente, seguindo método apresentado por Camargo et al. (1986), e quimicamente seguindo método descrito por Raij et al. (1987), nos laboratórios do Departamento de Solos e Adubos da Faculdade de Ciências Agrárias e Veterinárias da Universidade Estadual Paulista, Campus de Jaboticabal. A comparação das áreas foi feita pela análise de variância e pelo teste $\mathrm{F}$.

\section{RESULTADOS E DISCUSSÃO}

A comparação dos atributos físicos mostra que há pouca diferença entre o solo do SAF e o da floresta na mesma propriedade (Quadro 2). Os resultados da análise de variância também indicam que não há diferença significativa quanto à composição textural (Quadro 3) entre os solos dos sistemas agroflorestais e os das florestas remanescentes adjacentes, o que sugere a hipótese de tratar-se, em cada propriedade, do mesmo tipo de solo. Resultado semelhante foi verificado por Mcgrath et al. (2001), que constataram não haver diferença entre as frações do tamanho das partículas de um solo sob sistema agroflorestal de seis anos e aquelas encontradas na floresta nativa adjacente em pesquisa realizada no município de Nova Califórnia, no Estado de Rondônia. Isto evidencia que as propriedades físicas dos solos não foram alteradas após cinco anos e meio de uso com sistemas agroflorestais, o que era esperado já que não ocorreu erosão e o solo não foi revolvido (gradeado ou arado). Conseqüentemente, as diferenças nas características químicas (Quadros 4 e 5) devem ser atribuídas, principalmente, às atividades agrícolas, como capina, queimada e limpeza da área utilizada com sistemas agroflorestais.

$\mathrm{O}$ teste $\mathrm{F}$ da análise de variância indica que os valores de $\mathrm{pH}$, Ca e Mg diferem-se significativamente entre os solos dos sistemas agroflorestais e os das 
Quadro 2. Análise granulométrica de solos sob sistemas agroflorestais (SAF) e florestas remanescentes adjacentes (FLOR) em sete localidades na região de Itapuã do Oeste, RO

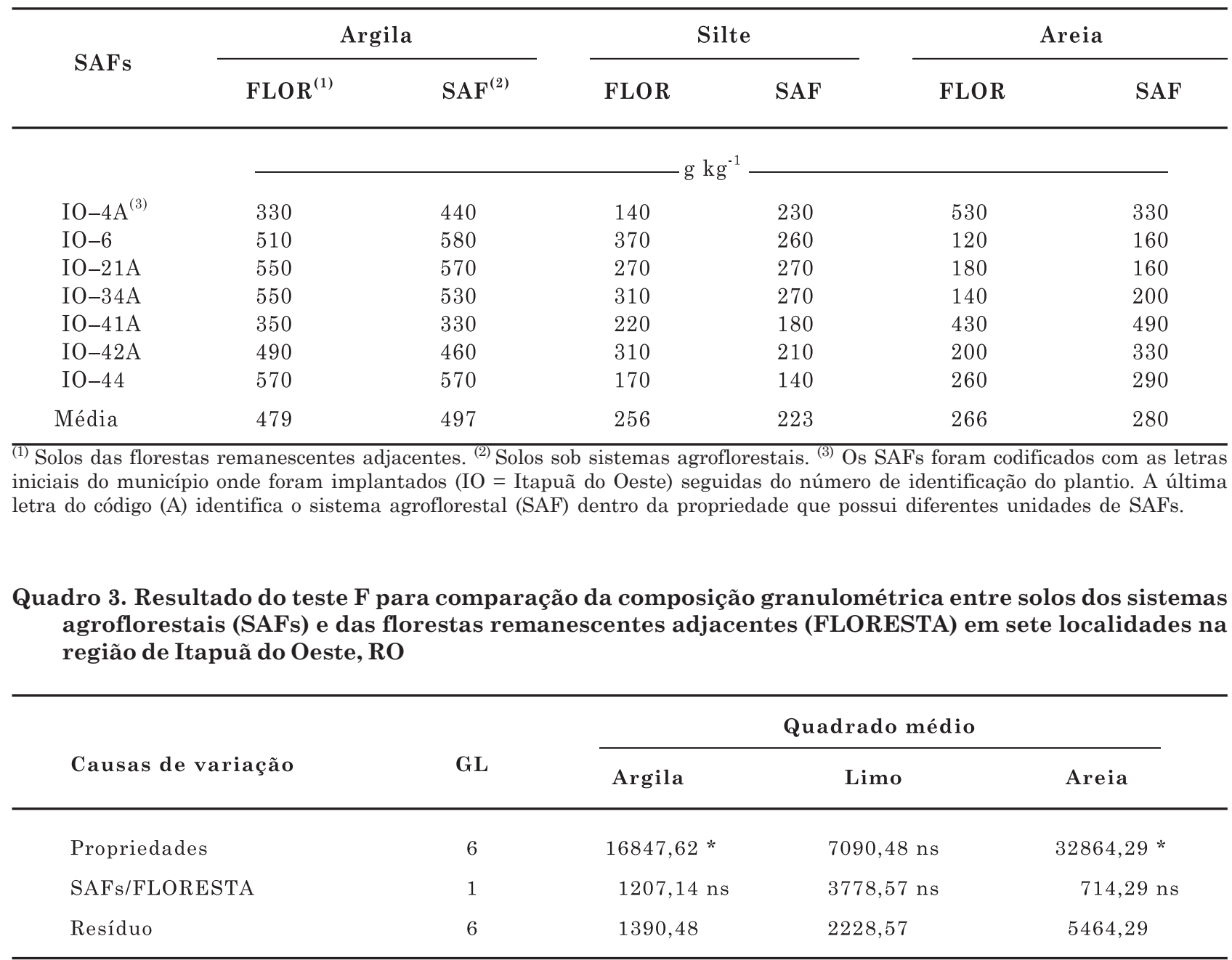

ns: não-significativo $(\mathrm{p}>0,05) ; *$ : significativo $(\mathrm{p}<0,05)$.

Quadro 4. Características químicas de solos sob sistemas agroflorestais (SAF) e florestas remanescentes adjacentes (FLOR) em sete localidades na região de Itapuã do Oeste, RO, em agosto de 2001

\begin{tabular}{|c|c|c|c|c|c|c|c|c|c|c|c|c|}
\hline \multirow{2}{*}{ SAFs } & \multicolumn{2}{|c|}{$\mathbf{P}$} & \multicolumn{2}{|c|}{ MO } & \multicolumn{2}{|c|}{ pH $\mathrm{CaCl}_{2}$} & \multicolumn{2}{|c|}{$\mathbf{K}^{+}$} & \multicolumn{2}{|c|}{$\mathrm{Ca}^{2+}$} & \multicolumn{2}{|c|}{$\mathrm{Mg}^{2+}$} \\
\hline & FLOR & SAF & FLOR & SAF & FLOR & SAF & FLOR & SAF & FLOR & SAF & FLOR & SAF \\
\hline & \multicolumn{2}{|c|}{$-\mathrm{mg} \mathrm{dm}^{-3}$} & \multicolumn{2}{|c|}{$-\mathrm{g} \mathrm{dm}^{-3}-$} & & & & & \multicolumn{2}{|c|}{$-\mathrm{mmol}_{\mathrm{c}} \mathrm{dm}^{-3}$} & & \\
\hline $\mathrm{IO}-4 \mathrm{~A}^{(1)}$ & 9 & 8 & 36 & 36 & 3,7 & 5,1 & 1,1 & 1,5 & 3 & 37 & 2 & 8 \\
\hline IO-6 & 8 & 7 & 41 & 50 & 4,4 & 5,2 & 1,1 & 1,6 & 14 & 38 & 5 & 11 \\
\hline IO-21A & 8 & 8 & 42 & 38 & 3,7 & 3,8 & 1,1 & 1,2 & 1 & 5 & 1 & 3 \\
\hline $\mathrm{IO}-34 \mathrm{~A}$ & 7 & 8 & 38 & 34 & 3,8 & 4,2 & 1,0 & 1,6 & 7 & 18 & 3 & 5 \\
\hline $\mathrm{IO}-41 \mathrm{~A}$ & 9 & 6 & 30 & 33 & 3,7 & 3,9 & 1,1 & 0,8 & 1 & 5 & 1 & 2 \\
\hline $\mathrm{IO}-42 \mathrm{~A}$ & 6 & 6 & 34 & 34 & 3,8 & 4,0 & 0,8 & 1,2 & 1 & 10 & 1 & 3 \\
\hline IO-44 & 6 & 7 & 29 & 31 & 3,9 & 4,0 & 1,3 & 1,0 & 2 & 9 & 1 & 2 \\
\hline Média & 8 & 7 & 36 & 37 & 3,9 & 4,3 & 1,1 & 1,3 & 4 & 17 & 2 & 5 \\
\hline
\end{tabular}

(1) Os SAFs foram codificados com as letras iniciais do município onde foram implantados (IO = Itapuã do Oeste) seguidas do número de identificação do plantio. A última letra do código (A) identifica o sistema agroflorestal (SAF) dentro da propriedade que possui diferentes unidades de SAFs. 
florestas remanescentes adjacentes (Quadro 5). Nos solos dos $\mathrm{SAFs}$, os valores de $\mathrm{pH}$, Ca e Mg foram sempre maiores que os dos solos das florestas remanescentes adjacentes (Quadro 4).

O aumento dos valores de $\mathrm{pH}$, e dos teores de Ca e Mg devem-se, provavelmente, ao aporte de nutrientes proveniente da queimada da floresta nativa por ocasião da abertura do terreno para uso agrícola. Verifica-se que, no $\mathrm{SAF}$, o teor de Ca do solo, em média, foi quatro vezes maior do que o do solo da floresta remanescente adjacente. Resultado semelhante foi relatado por Mcgrath et al. (2001), que observaram elevação de aproximadamente quatro vezes no teor de Ca no solo sob sistema agroflorestal de seis anos em relação ao da floresta remanescente adjacente no Estado de Rondônia.

Como não houve diferença significativa entre os teores de $\mathrm{P}$ e de $\mathrm{K}$ dos solos dos SAFs e das florestas remanescentes adjacentes (Quadro 5), esses nutrientes, liberados após a queimada ocorrida entre 6,5 e 14,5 anos antes da amostragem do solo, podem ter sido exportados com os produtos agrícolas obtidos no local ou absorvidos pelas árvores do SAF. Essa hipótese é reforçada por Alfaia et al. (2004), quando mencionam a possível exportação desses nutrientes por sucessivas safras de produtos em SAFs avaliados em Nova Califórnia/Rondônia. É possível, também, que parte do K liberado tenha se perdido por lixiviação e o $\mathrm{P}$ transformado em forma não disponível para as plantas.

Vários estudos reportam as mudanças que as queimadas causam nas propriedades químicas do solo logo após a retirada da floresta (Sanchez et al., 1982; Brady, 1996; Palm et al., 1996; Serrão et al., 1996). Os diferentes usos e manejos do solo podem interferir na manutenção dessas mudanças. A maioria dos trabalhos relacionados com esse tema menciona que os nutrientes disponibilizados pelas queimadas das florestas nativas na Amazônia usualmente suportam de dois a três anos com cultivos anuais. Após este período, a produtividade desses cultivos decai drasticamente, obrigando os produtores a abandonarem as áreas para pousio (Dubois at al., 1996).

Confirmando observações feitas por Mcgrath et al. (2001), este estudo indica que as mudanças no manejo do solo, resultantes da queima e conversão da floresta para um sistema agroflorestal, podem manter ou até mesmo melhorar algumas propriedades químicas do solo da floresta natural por pelo menos cinco anos e meio.

Não foi constatada diferença significativa entre o conteúdo de matéria orgânica do SAF e o da floresta adjacente (Quadro 5). Isso confirma os dados de Palm et al. (2004), de que os valores de C encontrado na camada de $0-20 \mathrm{~cm}$ do solo sob SAFs correspondem a $80-100 \%$ do valor desse elemento na floresta. Neste aspecto, o SAF se distingue favoravelmente de sistemas de culturas anuais e dos pousios de curta duração, que têm em seu solo apenas 50 e $65 \%$, respectivamente, do $\mathrm{C}$ encontrado no solo da floresta (Palm et al., 2004). Manter um alto teor de matéria orgânica no solo é de grande interesse para suas qualidades produtivas, uma vez que isso aumenta a capacidade retenção de água e de nutrientes, além de contribuir para a melhoria da estrutura do solo.

\section{CONCLUSÕES}

1. A comparação entre os atributos físicos (textura) do solo sob sistemas agroflorestais e os das florestas remanescentes adjacentes, evidenciou que não houve diferenças notáveis entre os solos comparados.

2. Verificou-se que o $\mathrm{pH}$ e os teores de $\mathrm{Ca}^{2+}{\mathrm{e} \mathrm{Mg}^{2+}}^{2+}$ foram mais altos nos plantios agroflorestais do que nos solos das florestas remanescentes adjacentes.

3. Os teores de matéria orgânica, P e K no solo não diferiram nos dois sistemas, indicando que os sistemas agroflorestais preservaram algumas propriedades químicas do solo com valores similares ao da floresta natural durante o período considerado.

Quadro 5. Resultado do teste F para comparação da composição química entre solos dos sistemas agroflorestais (SAFs) e das florestas remanescentes adjacentes (FLORESTA) em sete localidades na região de Itapuã do Oeste, $\mathrm{RO}$

\begin{tabular}{lcccccccc}
\hline & & \multicolumn{5}{c}{ Quadrado médio } \\
\cline { 6 - 9 } Causas de variação & GL & $\mathbf{P}$ & MO & $\mathbf{p H}$ & $\mathbf{K}^{+}$ & $\mathbf{C a}^{2+}$ & $\mathbf{M g}^{2+}$ \\
\hline Propriedades & 6 & $1,4524 \mathrm{~ns}$ & $55,4524^{*}$ & $0,2879 \mathrm{~ns}$ & $0,0481 \mathrm{~ns}$ & $165,4762 \mathrm{~ns}$ & $11,7381^{*}$ \\
SAFs/FLORESTA & 1 & $0,6429 \mathrm{~ns}$ & $2,5714 \mathrm{~ns}$ & $0,7314^{*}$ & $0,1400 \mathrm{~ns}$ & $617,7857^{*}$ & $28,5714^{*}$ \\
Resíduo & 6 & 0,9762 & 10,0714 & 0,1164 & 0,0700 & 64,9524 & 2,4048 \\
\hline
\end{tabular}

ns: não-significativo $(\mathrm{p}>0,05) ;{ }^{*}$ : significativo $(\mathrm{p}<0,05)$. 


\section{LITERATURA CITADA}

ALFAIA, S.S.; RIBEIRO, G.A.; NOBRE, A.D.; LUIZÃO, R.C. \& LUIZÃO, F.J. Evaluation of soil fertility in smallholder agroforestry systems and pastures in Western Amazônia. Agric. Ecosyst. Environ., 102:409-414, 2004.

BASTOS, T.X. \& DINIZ, T.D.A.S. Avaliação do clima do Estado de Rondônia para desenvolvimento agrícola. Belém, Embrapa-CPATU, 1982. 28p. (Boletim de Pesquisa, 44)

BRADY, N.C. Alternatives to slash-and-burn: A global imperative. Agric. Ecosyst. Environ., 58:3-1, 1996.

CAMARGO, O.A.; MUNIZ, A.C.; JORGE, J.A. \& VALADARES, J.M.A.S. Método de análise química, mineralógica e física de solo do Instituto Agronômico de Campinas. Campinas, Instituto Agronômico de Campinas, 1986. 94p. (Boletim Técnico, 106)

DUBOIS, J.C.L.; VIANA, V.M. \& ANDERSON, A.B. Sistemas e práticas agroflorestais para a Amazônia. Manual agroflorestal para a Amazônia. Rio de Janeiro, REBRAF, 1996. v.1. p.2-27.

McGRATH, D.A.; COMENFORD, N.B. \& DURYEA, M.L. Litter dynamics and monthly fluctuations in soil phosphorus availability in Amazonian agroforestry. For. Ecol. Manag., 131:p.167-184, 2000.

McGRATH, D.A.; DURYEA, M.L. \& CROPPER, W.P. Soil phosphorus availability and fine root proliferation in Amazonian agroforests 6 years following forest conversion. Agric. Ecosyst. Environ., 83:271-284, 2001.
PALM, A.C.; SWIFT, M.J. \& WOOMER, P.L. Soil biological dynamics in slash-and-burn agriculture. Agric. Ecosyst. Environ., 58:61-74, 1996.

PALM, C.; TOMICH, T.; van NOORDWIJK, M.; VOSTI, S.; GOCKOWSKI, J.; JULIO ALEGRE, J. \& VERCHOT, L. Mitigating GHG emissions in the humid tropics: Case studies from the alternatives to slash-and-burn program (ASB). Environ., Develop. Sustainab., 6:145-162, 2004.

RAIJ, B.van; QUAGGIO, J.A.; CANTARELLA, H.; FERREIRA, M.E.; LOPES, A.S. \& BATAGLIA, O.C. Análise química do solo para fins de fertilidade. Campinas, Fundação Cargill, 1987. 170p.

SANCHEZ, P.A.; BANDY, D.E.; VILLACHICA, J.H. \& NICHOLAIDES, J.J. Amazon basin soils: Management for continuous crop production. Science, 216:821-827, 1982.

SERRÃO, E.A.S.; NEPSTAD, D. \& WALKER, R. Upland agricultural and forestry development in the Amazon: Sustainability, criticality and resilience. Ecol. Econ., 18:313, 1996.

SMITH, N.; DUBOIS, J.; CURRENT, D. \& CLEMENT, C. Experiências agroflorestais na Amazônia Brasileira: restrições e oportunidades. Brasília, Banco Mundial, 1998. $120 \mathrm{p}$.

van LEEUWEN, J. Desenvolvimento e avaliação participativa de sistemas agroflorestais. Brasília, 2002. p.88-93,196. Disponível em: <http://www.inpa.gov.br/cpca/ johannes/ livro-PPD-2000-2003.pdf>. Acesso em: 18 de ago. de 2004. 